SFB

An order for asymmetry in copulas, and implications for risk management

Karl Friedrich Siburg, Katharina Stehling,

Pavel A. Stoimenov, Jeannette H. C. Woerner

Nr. 44/2013

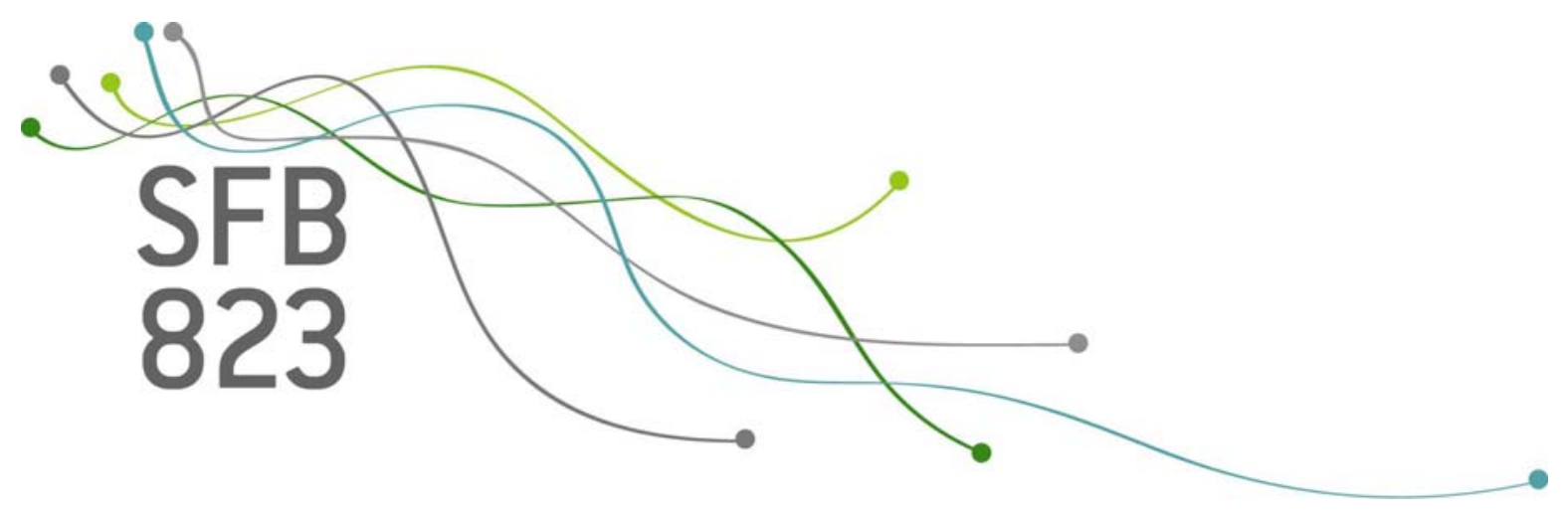





\title{
An order for asymmetry in copulas, and implications for risk management
}

\author{
Karl Friedrich Siburg \\ Fakultät für Mathematik \\ Technische Universität Dortmund \\ Katharina Stehling \\ Wirtschafts- und Sozialwissenschaftliche Fakultät \\ Technische Universität Dortmund
}

\author{
Pavel A. Stoimenov \\ International Energy Company \\ Wien
}

\author{
Jeannette H. C. Woerner \\ Fakultät für Mathematik \\ Technische Universität Dortmund
}

November 13, 2013

\begin{abstract}
We investigate symmetry properties of bivariate copulas. For this, we introduce an order of asymmetry, as well as measures of asymmetry which are monotone in that order. As for applications, we show that asymmetry does occur in real financial data. This implies that in finance and risk management, asymmetric models should be favored against the usual symmetric ones.
\end{abstract}

\section{Introduction and results}

Modelling the dependence structure between random variables is essential for finance and risk management. In practice, this leads to the problem of fitting a copula to a given set of data, which is mostly addressed by choosing an 'appropriate' parametric family of bivariate copulas and finding the 'correct' parameter. Obviously, the choice of the copula family determines the goodness of the fit and the predictions in a fundamental way. Nowadays, the practitioner has a variety of parametric copula families at hand, the most prominent of which are Archimedean copulas.

However, all of these families are unable to incorporate a fundamental feature of the data and, hence, are not optimal for applications. This fundamental feature is asymmetry, by which we mean the fact that $C(u, v)$ may not be the same as 
$C(v, u){ }^{1}$ All standard copula models, in particular all Archimedean copulas, are symmetric, i.e., they satisfy $C(u, v)=C(v, u)$ for all $u, v$. Therefore, if a given set of data possesses some degree of asymmetry, these standard models are not adequate, and asymmetric copula models should be taken into consideration.

Asymmetry of copulas has been considered, for instance, in [KM, Ne2] where the copulas with the largest measure of asymmetry are identified. However, without an underlying order of asymmetry, results of this kind can be misleading; see Rem. 3.19). Only recently symmetry and asymmetry of copulas have been considered from a statistical point of view. [GNQ] constructed a test of global symmetry and applied it to nutrition data, whereas $[\mathrm{KY}]$ only considered the special case of extreme value copulas and applied their test to insurance data.

The aim of this paper is twofold. First, we want to develop a consistent theory of asymmetry in bivariate distributions. In order to be able to quantify the degree of asymmetry of a copula, we introduce an order of asymmetry for copulas and define several measures which are monotone in that order. It is important to understand that measures without an underlying order are not sufficient for comparing the degree of asymmetry of two given copulas. Indeed, one might be tempted to call a copula $C$ less asymmetric than another copula $D$ provided $\mu(C) \leq \mu(D)$ for some reasonable asymmetry measure $\mu$. However, there will often exist other, equally reasonable measures of asymmetry satisfying the reverse inequality. If, on the other hand, we can define an order $\prec$ of asymmetry we know a copula $C$ is less asymmetric than $D$ if, and only if, $C \prec D$. In this case, all measures $\mu$ that are monotone in the order $\prec$ (i.e., $\mu(C) \leq \mu(D)$ whenever $C \prec D$ ) are consistent with the concept of asymmetry and do not lead to misinterpretations. Of course, all said applies equally well to any other situation where one wants to quantify a certain feature - the use of measures without an underlying order is prone to creating mistakes.

The second goal of our paper is to show that asymmetry does indeed occur in empirical financial data. Based on central limit theorem for the empirical copula we provide results indicating whether $C(u, v)=C(v, u)$ for each individual point $(u, v)$. In contrast to [GNQ] this yields a precise picture in which regions the asymmetry of a copula is most prominent.

The paper is organized as follows. In Section 2 we present a short introduction to the concept of copulas and gather the results relevant for our purposes. In the central Section 3, we introduce an order of asymmetry, investigate its properties, and construct several measures of asymmetry monotone in that order. The final Section 4 illustrates that asymmetry occurs in different sets of financial data.

Acknowledgements. The authors thank Alexander Paduch for programming the empirical examples. JHCW gratefully acknowledges financial support by the Deutsche Forschungsgemeinschaft (SFB 823, Project C5).

\footnotetext{
${ }^{1}$ Note that the term asymmetry is also used in a different context, namely when referring to the different behavior of the lower and upper tail dependence.
} 


\section{Preliminaries}

Consider two real-valued random variables $X$ and $Y$ on some probability space. Then $X$ and $Y$ are called exchangeable if $(X, Y)$ and $(Y, X)$ have the same distribution, i.e. if their joint distribution function $F_{X, Y}$ is symmetric:

$$
F_{X, Y}(x, y)=F_{X, Y}(y, x) \text {. }
$$

Note that exchangeable random variables are necessarily identically distributed.

Let us briefly recall the basic concepts of the theory of copulas; for details we refer to $[\mathrm{Ne} 1]$. Let $I=[0,1]$ be the compact unit interval. A (bivariate) copula is a function $C: I^{2} \rightarrow I$ with the following properties:

1. $C(u, 0)=C(0, v)=0$ for all $u, v \in I$.

2. $C(u, 1)=u$ and $C(1, v)=v$ for all $u, v \in I$.

3. $C\left(u_{2}, v_{2}\right)-C\left(u_{2}, v_{1}\right)-C\left(u_{1}, v_{2}\right)+C\left(u_{1}, v_{1}\right) \geq 0$ for all $\left[u_{1}, u_{2}\right] \times\left[v_{1}, v_{2}\right] \subseteq I^{2}$.

Given two real-valued random variables $X$ and $Y$ with joint distribution function $F_{X, Y}$ and univariate margins $F_{X}$ and $F_{Y}$, respectively, a theorem by Sklar states that there exists a copula $C_{X, Y}$ such that

$$
F_{X, Y}(x, y)=C_{X, Y}\left(F_{X}(x), F_{Y}(y)\right) .
$$

Moreover, if $F_{X}$ and $F_{Y}$ are continuous the function $C_{X, Y}$ is unique and will be referred to as the copula of $(X, Y)$.

It follows that, for continuous identically distributed random variables $X$ and $Y$, we have $C_{Y, X}=C_{X, Y}^{\top}$ where the copula $C^{\top}$ is defined by

$$
C^{\top}(u, v):=C(v, u)
$$

Therefore, exchangeability of two identically distributed random variables $X$ and $Y$ is equivalent to the symmetry of their copula $C_{X, Y}$ :

$$
C_{X, Y}=C_{X, Y}^{\top}
$$

There are three distinguished copulas, corresponding to three distinguished dependence structures of $X$ and $Y$, namely the independence copula $P(u, v)=u v$ (where $X$ and $Y$ are independent) as well as the Fréchet-Hoeffding bounds $W(u, v)=$ $\max (u+v-1,0)$ and $M(u, v)=\min (u, v)$ (where $Y=f(X)$ for some monotone increasing, respectively decreasing, function $f)$. It is easily shown that

$$
W(u, v) \leq C(u, v) \leq M(u, v)
$$

for every copula $C$.

Given a copula $C$, its survival copula $\hat{C}$ is defined by

$$
\hat{C}(u, v):=u+v-1+C(1-u, 1-v) .
$$

If $C$ corresponds to the distribution function $F_{X, Y}$, its survival copula corresponds to the joint survival function $\hat{F}(x, y)$ given by $\hat{F}(x, y)=1-F_{X}(x)-F_{Y}(y)+F_{X, Y}(x, y)$. 
Finally, it follows immediately from the definition that the set of copulas is convex, i.e., if we have two copulas $C_{0}$ and $C_{1}$ then the function

$$
C_{t}:=(1-t) C_{0}+t C_{1}=C_{0}+t\left(C_{1}-C_{0}\right)
$$

is also a copula for each $t \in I$.

\section{An order and nonparametric measures of asymmetry}

\subsection{An order of asymmetry for copulas}

Definition 3.1. A copula $C$ is called symmetric if $C=C^{\top}$, and asymmetric otherwise.

Definition 3.2. A copula $C$ is said to be less asymmetric than a copula $D$, written $C \prec D$, if and only if

$$
|C(u, v)-C(v, u)| \leq|D(u, v)-D(v, u)|
$$

for all $(u, v) \in I^{2}$. We call $\prec$ the order of asymmetry on the set of copulas.

Remark 3.3. 1. Note that $C \prec D$ is the same as saying that

$$
\left|C-C^{\top}\right| \leq\left|D-D^{\top}\right|
$$

pointwise in $I^{2}$.

2. It is easy to see that the relation $\prec$ is reflexive and transitive. However, it is not antisymmetric since $C \prec D$ and $D \prec C$ is equivalent to $\left|C-C^{\top}\right|=\left|D-D^{\top}\right|$ which does not imply that $C=D$ (consider, for instance, symmetric $C$ and $D)$. Therefore, $\prec$ is a preorder and not an order; nevertheless we will use the term 'order of asymmetry' for $\prec$.

3. $\prec$ is not total, i.e., there are copulas which cannot be ordered w.r.t. $\prec$.

Definition 3.4. Consider a set $S$ with a preorder $\leq$.

An element $m \in S$ is called a maximal element if $x \leq m$ for all $x \in S$ which are comparable to $m$ (i.e., which satisfy $x \leq m$ or $m \leq x$ ). An element $m \in S$ is called $a$ greatest element if $x \leq m$ for all $x \in S$.

Analogously, one defines minimal, respectively smallest, elements by replacing $\leq$ by $\geq$.

Remark 3.5. It is clear that any greatest element is also maximal; the converse, in general, false.

Note that, since we are dealing with preorders instead of orders, there may be more than one greatest element. Note that $C \prec D \prec C$ just means that $\left|C-C^{\top}\right|=$ $\left|D-D^{\top}\right|$ everywhere, which does not mean $C=D$ (consider, for instance, $D=C^{\top}$ for an asymmetric $C$ ). 
The following results state, loosely speaking, that the symmetric copulas are precisely the least asymmetric copulas, but that there is no maximally asymmetric copula.

Proposition 3.6. Each symmetric copula is a smallest (hence a minimal) element w.r.t. $\prec$, and there are no other minimal elements.

Proof. The first assertion follows from the fact that each symmetric copula $C$ is comparable to any other copula $D$, and satisfies $C \prec D$. The fact that any minimal element must be comparable to all symmetric copulas implies that any minimal element must be symmetric itself.

Theorem 3.7. There is no greatest element w.r.t. $\prec$.

Proof. Klement and Mesiar $[\mathrm{KM}]$ have shown that for any copula $C$ we have

$$
0 \leq|C(u, v)-C(v, u)| \leq \min (u, v, 1-u, 1-v,|u-v|)
$$

for every $(u, v) \in I^{2}$, and that for each $(u, v) \in I^{2}$ there exists a copula $C$ such that $|C(u, v)-C(v, u)|=\min (u, v, 1-u, 1-v,|u-v|)$. Hence, if $D$ is a greatest element w.r.t. $\prec$ we have $|D(u, v)-D(v, u)|=\min (u, v, 1-u, 1-v,|u-v|)$ for every $(u, v) \in I^{2}$. But it is also proven in $[\mathrm{KM}]$ that there does not exist a copula $D$ with the above property.

The next result states that $\prec$ is invariant under transposition and survival operation.

Proposition 3.8. Let $C, D$ be two copulas with $C \prec D$. Then the transpose and the survival copulas satisfy the same relation, i.e.

$$
C^{\top} \prec D^{\top} \text { and } \hat{C} \prec \hat{D} \text {. }
$$

Proof. This follows directly from the definitions.

\subsection{Constructing ordered families of copulas}

Almost all copulas dealt with in application are symmetric, e.g., all Archimedean copulas. In order to construct asymmetric copulas, one can apply general methods like gluing [SS], patchwork constructions [DuSS], or a method mentioned in [GKNY].

In the following we will construct one-parameter families of copulas that are ordered w.r.t. $\prec$. Note, however, that constructing asymmetric copulas is more complicated than constructing symmetric ones.

As a first class of examples, let us investigate convex combinations. So, given any two copulas $C_{0}$ and $C_{1}$, consider the family of copulas

$$
C_{t}=C_{0}+t\left(C_{1}-C_{0}\right)
$$

for $t \in I$. When is this family ordered w.r.t. $\prec$ ? In order to investigate this question, let us define the functions $d_{0}(u, v):=\left(C_{0}-C_{0}^{\top}\right)(u, v)$ and $d_{1}(u, v):=\left(C_{1}-C_{1}^{\top}\right)(u, v)$, as well as

$$
d_{t}(u, v):=d_{0}(u, v)+t\left(d_{1}-d_{0}\right)(u, v)
$$


where $(u, v) \in I^{2}$ and $t \in I$. Then we have

$$
C_{s} \prec C_{t} \Longleftrightarrow\left|d_{s}(u, v)\right| \leq\left|d_{t}(u, v)\right| \text { for all }(u, v) \in I^{2} .
$$

Theorem 3.9. If $d_{t}(u, v)$ has a fixed sign for all $(u, v) \in I^{2}$ and all $t \in I$, and $\left(d_{1}-d_{0}\right)(u, v)$ has a fixed sign for all $(u, v) \in I^{2}$, then the family $C_{t}, t \in I$, is ordered w.r.t. $\prec$.

Proof. For every fixed $(u, v) \in I^{2}$, the function $t \mapsto d_{t}(u, v)$ is affine, hence monotone. Since $d_{1}-d_{0}$ has a fixed sign for all $(u, v), t \mapsto d_{t}(u, v)$ is either monotonically increasing for all $(u, v)$, or monotonically decreasing for all $(u, v)$. Therefore, as long as $d_{t}(u, v) \neq 0$, the function $t \mapsto\left|d_{t}(u, v)\right|$ has the same monotonicity behavior.

Theorem 3.10. If $C_{0}$ and $C_{1}$ are two copulas such that $C_{0}$ is symmetric, then the family $C_{t}, t \in I$, is ordered w.r.t. $\prec$. More precisely, we have $C_{s} \prec C_{t}$ whenever $s \leq t$

Proof. The assumption on $C_{0}$ is tantamount to $d_{0}=0$ everywhere on $I^{2}$ so that $\left|d_{t}\right|=t\left|d_{1}\right|$, and the assertion follows.

The following example shows that the order of asymmetry is not compatible with the stochastic order on copulas, where one requires that $C \leq D$ pointwise on $I^{2}$.

Example 3.11. Consider the case where $C_{0}=W$ is the lower Fréchet-Hoeffding bound, and $C_{1}=C$ is any asymmetric copula. Then the family

$$
C_{t}=W+t(C-W), \quad t \in I,
$$

is ordered w.r.t. $\prec$, as well as w.r.t. the stochastic order. More precisely, we have $C_{s} \prec C_{t}$ and $C_{s} \leq C_{t}$ whenever $s \leq t$.

Similarly, if $C_{0}=M$, then the family

$$
D_{t}=M+t(C-M), \quad t \in I,
$$

is also ordered w.r.t. both orders. Note, however, that the stochastic ordering is in the negative direction, i.e., we still have $D_{s} \prec D_{t}$ whenever $s \leq t$, but now $D_{s} \geq D_{t}$.

In the following, we turn our attention to more elaborate examples. If $C$ is a symmetric copula (e.g., any Archimedean copula) and $\alpha, \beta \in(0,1)$ with $\alpha \neq \beta$, then it is shown in [GKNY] that the two-parameter family

$$
C_{\alpha, \beta}(u, v):=u^{1-\alpha} v^{1-\beta} C\left(u^{\alpha}, v^{\beta}\right)
$$

consists of asymmetric copulas. In particular, if $\beta=1-\alpha$, we obtain the oneparameter family

$$
C_{\alpha}(u, v)=u^{1-\alpha} v^{\alpha} C\left(u^{\alpha}, v^{1-\alpha}\right)
$$

consisting of asymmetric copulas (as long as $\alpha \neq 1 / 2$ ). 
Proposition 3.12. Let $C$ be a symmetric copula, and consider the family $C_{\alpha}$ as given in (5). Then

$$
C_{\alpha} \prec C_{1-\alpha}
$$

for all $\alpha \in(0,1)$.

Proof. This follows immediately from the identities $C_{\alpha}(u, v)=C_{1-\alpha}(v, u)$ and $C_{\alpha}(v, u)=C_{1-\alpha}(u, v)$, so that

$$
\left|\left(C_{\alpha}-C_{\alpha}^{\top}\right)(u, v)\right|=\left|\left(C_{1-\alpha}-C_{1-\alpha}^{\top}\right)(u, v)\right|
$$

for all $(u, v) \in I^{2}$.

Remark 3.13. Note that, by symmetry in $\alpha$, we also have that $C_{1-\alpha} \prec C_{\alpha}$. Since $\prec$ is not antisymmetric, this is not a contradiction to the fact that $C_{\alpha} \neq C_{1-\alpha}$.

In his work on maximally asymmetric copulas [Ne2], Nelsen studies the twoparameter family of copulas given by

$$
N_{s, t}(u, v):=u v+u v(1-u)(1-v)(s+(t-s) v(1-u))
$$

where $s \in[-1,1]$ and $\left(s-3-\sqrt{9+6 s-3 s^{2}}\right) / 2 \leq t \leq 1$.

Theorem 3.14. Fix some $s \in[-1,1]$. Then

$$
N_{s, t_{1}} \prec N_{s, t_{2}}
$$

for any $t_{1}, t_{2}$ such that $\left|t_{1}-s\right| \leq\left|t_{2}-s\right|$.

Proof. We have to show that $\left|N_{s, t_{1}}-N_{s, t_{1}}^{\top}\right| \leq\left|N_{s, t_{2}}-N_{s, t_{2}}^{\top}\right|$ pointwise on $I^{2}$. But since

$$
\left|\left(N_{s, t}-N_{s, t}^{\top}\right)(u, v)\right|=u v(1-u)(1-v)|v-u| \cdot|t-s|,
$$

this is equivalent to $\left|t_{1}-s\right| \leq\left|t_{2}-s\right|$.

For the following, let $K$ be the copula defined by

$$
K(u, v):=u v-u^{3} v(1-u)(1-v) .
$$

We want to compare it to copulas from the family $N_{s, t}$.

Theorem 3.15. We have

$$
K \prec N_{s, t} \Longleftrightarrow|s-t| \geq 2 .
$$

Proof. By a simple calculation we see that

$$
\left|\left(K-K^{\top}\right)(u, v)\right|=u v(1-u)(1-v)|v-u| \cdot|u+v| .
$$

In view of (6), we conclude that $K \prec N_{s, t}$ if, and only if, $|u+v| \leq|t-s|$ for all $(u, v) \in I^{2}$, and the claim follows.

Example 3.16. Setting $s=0$ and $t=-3$, for instance, we see that

$$
u v-u^{3} v(1-u)(1-v) \prec u v-3 u v^{2}(1-u)^{2}(1-v) .
$$




\subsection{Monotone measures of asymmetry for copulas}

Having introduced an order of asymmetry $\prec$ for copulas, it is natural to look for measures of asymmetry which are monotone with respect to that order.

Definition 3.17. A nonnegative function $\mu$ defined on the set of bivariate copulas is a monotone measure of asymmetry if it satisfies the following two conditions:

1. $\mu(C)=0$ if and only if $C$ is symmetric.

2. $C \prec D$ implies $\mu(C) \leq \mu(D)$.

Remark 3.18. Note that $\mu(C)=0$ is equivalent to $C$ being symmetric. This is much stronger a requirement than just asking for $\mu(C)=0$ whenever $C$ is symmetric.

Remark 3.19. As mentioned in the introduction, it is of vital importance to have an order with respect to which the measures one considers are monotone. 'Ordering by measure', i.e. ordering without an order, necessarily leads to misinterpretations, as shall be illustrated by the following simple example.

Assume you want to decide when a function $f: I \rightarrow[0, \infty)$ is "bigger" than another function $g: I \rightarrow[0, \infty)$. There are at least two equally reasonable ways to do so, namely by comparing either their maximal value (i.e. taking $\|f\|_{\infty}$ as a measure) or their mean (i.e. considering $\|f\|_{1}$ ). Now take the two functions $f(x)=1$ and $g(x)=2$ if $x \in[0,1 / 3]$ and $g(x)=0$ elsewhere. Which of them is bigger? The answer depends on the measure chosen.

This dilemma is resolved when one considers the obvious order- $f \prec g$ if and only if $f(x) \leq g(x)$ for all $x \in I$ - with respect to which both measures are monotone, but the two functions above are not comparable.

Note, by the way, that the term 'maximally nonexchangeable' [Ne2, KM] refers to such an ordering without an order.

Whereas the order of asymmetry describes a qualitative relation between the asymmetry of two copulas, a measure assigns a numerical value to a copula that expresses its degree of asymmetry in a quantitative way. The monotonicity condition guarantees that these two notions are compatible.

In this section, we introduce monotone $L^{p}$-measures of asymmetry for $p \in[1, \infty]$, and show how one can construct tailor-made monotone measures for various applications.

For each $p \in[1, \infty]$ we define

$$
\mu_{p}(C):=\left\|C-C^{\top}\right\|_{p},
$$

where $\|\cdot\|_{p}$ denotes the usual $L^{p}$-norm on continuous functions on $I^{2}$, i.e.,

$$
\|f\|_{p}=\left(\int_{I^{2}}|f(u, v)|^{p} d u d v\right)^{1 / p}
$$

for $p \in[1, \infty)$, and

$$
\|f\|_{\infty}=\max _{I^{2}}|f| .
$$


Theorem 3.20. For each $p \in[1, \infty]$, the function $\mu_{p}$ is a monotone measure of asymmetry.

Proof. This follows immediately from the definitions of $\mu_{p}$ and $\prec$.

Remark 3.21. The measure $\mu_{\infty}$ corresponds to the maximal degree of asymmetry a copula can attain in a point. For other aspects, however, other measures may be more relevant. For instance, if one is interested in the total amount of asymmetry of a copula, respectively its average degree of asymmetry, the measure $\mu_{1}$ should be studied instead.

One may also combine the monotone measures $\mu_{p}$ with different weights, say, and obtain new monotone measures of asymmetry, as the following result shows.

Theorem 3.22. Let $F:[0, \infty)^{k} \rightarrow[0, \infty)$ be monotonically increasing in each argument and satisfy $F>0$ on the interior of $[0, \infty)^{k}$ as well as $F(0, \ldots, 0)=0$. Pick any monotone measures of asymmetry $\mu_{i}$ for $1 \leq i \leq k$.

Then the composition $\mu:=F\left(\mu_{1}, \ldots, \mu_{k}\right)$ is also a monotone measure of asymmetry.

Proof. This follows readily from the definition of a monotone measure of asymmetry.

In particular, we may choose $\mu_{i}=\mu_{p_{i}}$ to be any of the $L^{p}$-measures introduced above, and obtain the following result.

Corollary 3.23. 1. Any linear combination $\sum_{i=1}^{k} m_{i} \mu_{p_{i}}$ with $m_{i}>0$ is a monotone measure of asymmetry.

2. Any product $\prod_{i=1}^{k} m_{i} \mu_{p_{i}}$ with $m_{i}>0$ is a monotone measure of asymmetry.

Finally, if one is interested in the asymmetry properties of copulas in a particular region of $I^{2}$ only, one can modify the definition of $\mu_{p}$ and incorporate an appropriate weight function. For instance, let $f: I \rightarrow[0, \infty)$ be given such that $f(u)>0$ for $u \in(0,1)$, and define a weight function $F: I^{2} \rightarrow[0, \infty)$ by $F(u, v):=f(u)$.

Theorem 3.24. Under the above assumptions, the function

$$
\mu_{p, F}(C):=\left\|\left(C-C^{\top}\right) \cdot F\right\|_{p}
$$

is a monotone measure of asymmetry.

Proof. If $C$ is symmetric then clearly $\mu_{p, F}(C)=0$; conversely, if $\mu_{p, F}(C)=0$ then $\left|C-C^{\top}\right|=0$, because $f(u)>0$ for all $u \in(0,1)$, and $C$ is symmetric. The monotonicity property is clear because of $F \geq 0$. 


\section{Application to financial data}

In this section, we will show that the phenomenon of asymmetry defined in Section 3.1 indeed occurs in financial data. Hence asymmetry is an aspect which should be included in a realistic model for dependencies - however, all the classical parametric families (e.g., Archimedean copulas) do not capture this feature.

The study of a nonparametric estimator of the copula, the empirical copula, and related convergence results under different conditions and in different metrics has a long history [De1, Rü, GS]. We will apply a result on the weak convergence of the empirical copula process by Fermanian et al. [FRW]. Before we state this theorem let us introduce some notation. Denote by

$$
F_{X, Y}^{n}(x, y):=\frac{1}{n} \sum_{i=1}^{n} 1_{X_{i} \leq x, Y_{i} \leq y}
$$

the empirical distribution function of the iid sequence $\left(X_{1}, Y_{1}\right), \cdots,\left(X_{n}, Y_{n}\right)$ for $x, y \in \mathbb{R}$. Let $F_{X}^{n}(x)=F_{X, Y}^{n}(x, \infty)$ and $F_{Y}^{n}(y)=F_{X, Y}^{n}(\infty, y)$ be the associated marginal distributions, then we define for $0 \leq u, v \leq 1$ the empirical copula function

$$
C_{X, Y}^{n}(u, v):=F_{X, Y}^{n}\left(\left(F_{X}^{n}\right)^{-}(u),\left(F_{Y}^{n}\right)^{-}(v)\right),
$$

where $F^{-}(u):=\inf \{t \mid F(t) \geq u\}$ denotes the generalized inverse of the distribution function $F$. Furthermore, by

$$
Z_{n}(u, v):=\sqrt{n}\left(C_{X, Y}^{n}-C_{X, Y}\right)(u, y)
$$

for $(u, v) \in I^{2}$ we denote the empirical copula process.

Theorem 4.1 ([FRW]). Suppose that $F_{X}$ and $F_{Y}$ are continuous and that $C$ has continuous partial derivatives. Then the empirical copula process $Z_{n}$ converges weakly in $L^{\infty}\left(I^{2}\right)$ to the Gaussian process $G_{C}$, where

$$
G_{C}(u, v):=B_{C}(u, v)-\frac{\partial}{\partial u} C(u, v) B_{C}(u, 1)-\frac{\partial}{\partial v} C(u, v) B_{C}(1, v),
$$

and $B_{C}(u, v)$ denotes a Brownian bridge on $I^{2}$ with covariance function

$$
E\left(B_{C}(u, v) B_{C}\left(u^{\prime}, v^{\prime}\right)\right)=C_{X, Y}\left(u \wedge u^{\prime}, v \wedge v^{\prime}\right)-C_{X, Y}(u, v) C_{X, Y}\left(u^{\prime}, v^{\prime}\right)
$$

for all $u, u^{\prime}, v, v^{\prime} \in I$.

From this theorem we can easily deduce consistency and asymptotic normality for the empirical copula.

Corollary 4.2. Under the conditions of Thm. 4.1, we obtain

$$
C_{X, Y}^{n}(u, v) \stackrel{p}{\rightarrow} C_{X, Y}(u, v)
$$

and

$$
\sqrt{n}\left(C_{X, Y}^{n}(u, v)-C_{X, Y}(u, v)\right) \stackrel{d}{\rightarrow} N(0, A)
$$


for all $(u, v) \in I^{2}$ as $n \rightarrow \infty$, where

$$
\begin{aligned}
A= & C_{X, Y}(u, v)\left(1-C_{X, Y}(u, v)\right)+\left(\frac{\partial}{\partial u} C_{X, Y}(u, v)\right)^{2} u(1-u)+\left(\frac{\partial}{\partial v} C_{X, Y}(u, v)\right)^{2} v(1-v) \\
& -2 \frac{\partial}{\partial u} C_{X, Y}(u, v) C_{X, Y}(u, v)(1-u)-2 \frac{\partial}{\partial v} C_{X, Y}(u, v) C_{X, Y}(u, v)(1-v) \\
& +2 \frac{\partial}{\partial u} C_{X, Y}(u, v) \frac{\partial}{\partial v} C_{X, Y}(u, v)\left(C_{X, Y}(u, v)-u v\right) .
\end{aligned}
$$

Proof. The consistency of $C_{X, Y}^{n}$ follows directly since $G_{C}$ is a zero mean Gaussian process by the properties of a Brownian bridge. For the asymptotic normality we only have to calculate

$$
\begin{aligned}
\operatorname{Var}\left(G_{C}(u, v)\right)= & E\left(G_{C}^{2}(u, v)\right) \\
= & E\left(B_{C}^{2}(u, v)\right)+\left(\frac{\partial}{\partial u} C_{X, Y}(u, v)\right)^{2} E\left(B_{C}^{2}(u, 1)\right) \\
& +\left(\frac{\partial}{\partial v} C_{X, Y}(u, v)\right)^{2} E\left(B_{C}^{2}(1, v)\right) \\
& -2 \frac{\partial}{\partial u} C_{X, Y}(u, v) E\left(B_{C}(u, v) B_{C}(u, 1)\right) \\
& -2 \frac{\partial}{\partial v} C_{X, Y}(u, v) E\left(B_{C}(u, v) B_{C}(1, v)\right) \\
& +2 \frac{\partial}{\partial u} C_{X, Y}(u, v) \frac{\partial}{\partial v} C_{X, Y}(u, v) E\left(B_{C}(u, 1) B_{C}(1, v)\right) \\
= & C_{X, Y}(u, v)\left(1-C_{X, Y}(u, v)\right)+\left(\frac{\partial}{\partial u} C_{X, Y}(u, v)\right)^{2} u(1-u) \\
& +\left(\frac{\partial}{\partial v} C_{X, Y}(u, v)\right)^{2} v(1-v) \\
& -2 \frac{\partial}{\partial u} C_{X, Y}(u, v) C_{X, Y}(u, v)(1-u) \\
& -2 \frac{\partial}{\partial v} C_{X, Y}(u, v) C_{X, Y}(u, v)(1-v) \\
& +2 \frac{\partial}{\partial u} C_{X, Y}(u, v) \frac{\partial}{\partial v} C_{X, Y}(u, v)\left(C_{X, Y}(u, v)-u v\right) .
\end{aligned}
$$

From this corollary we can deduce confidence intervals: the $95 \%$ confidence interval, for instance, is given by

$$
\left[C_{X, Y}^{n}(u, v)-\frac{1.96}{\sqrt{n}} \sqrt{A}, C_{X, Y}^{n}(u, v)+\frac{1.96}{\sqrt{n}} \sqrt{A}\right] .
$$

Hence, if $\left|C_{X, Y}^{n}(u, v)-C_{X, Y}^{n}(v, u)\right|>\frac{1.96}{\sqrt{n}} \sqrt{A}$, we have a probability of $95 \%$ for asymmetry in the point $(u, v)$. However, the asymptotic variance involves the unknown quantities $C_{X, Y}, \frac{\partial}{\partial u} C_{X, Y}(u, v)$ and $\frac{\partial}{\partial v} C_{X, Y}(u, v)$. We can make this confidence bounds feasible by plugging in consistent estimators for the quantities and use Slutzki's Lemma. As estimators for the copula we again use the empirical copula, 
whereas for the partial derivatives we use a kernel density estimator as in [DeSS], namely

$$
\frac{1}{n h_{1}} \sum_{i=1}^{n} K\left(\frac{u-F_{X}^{n}\left(X_{i}\right)}{h_{1}}\right) \bar{K}\left(\frac{v-F_{Y}^{n}\left(Y_{i}\right)}{h_{2}}\right) \stackrel{p}{\rightarrow} \frac{\partial}{\partial u} C_{X, Y}(u, v),
$$

where $K$ denotes a symmetric kernel with support $I, \bar{K}(x)=\int_{-1}^{x} K(t) d t$, and the bandwidths $h_{1}$ and $h_{2}$ are chosen such that $h_{1}, h_{2} \rightarrow 0, n h_{1}^{3} \rightarrow \infty, n h_{1} h_{2} \rightarrow \infty$, and $n h_{1}^{4} \rightarrow 0$. The partial derivative with respect to the second component is defined analogously.

As data sets we use daily log-returns of three indices: the S\&P 500, the VIX (volatility index on the S\&P 500), and the HUI (index based on assets of gold mining industry). In the following we consider the years 2006 and 2009 with $n=250$ observations. As bandwidths we choose $h_{1}=h_{2}=250^{-3 / 10}$, see also [DeSS], and as kernel we take the Epanechnikow kernel. We plot $\max \left(\left|C_{X, Y}^{n}(u, v)-C_{X, Y}^{n}(v, u)\right|-\right.$ $\left.1,96 \frac{\sqrt{A_{n}}}{\sqrt{n}}, 0\right)$, where $A_{n}$ denotes the estimated asymptotic variance. Hence positive values indicate asymmetry since the transposed entry $C_{X, Y}^{n}(v, u)$ lies outside the $95 \%$ confidence interval of $C_{X, Y}^{n}(u, v)$.

Figures 1 and 2 provide the results for the S\&P 500 and the VIX for the years 2006 and 2009, respectively. Note that we observe asymmetry in both years. Moreover, during the financial crisis the amount of asymmety increases substantially, especially leading to strong degree of asymmetry near the corners $(0,1)$ and $(1,0)$. Figures 3 and 4 provide the analogous results for the VIX and HUI, which are similar to the previous ones.

Finally, Figures 5 and 6 provide the results for the S\&P 500 and the HUI. Here the picture is different - the asymmetry seems to decrease, but is also located close the corner $(0,0)$, which provides information on the high risk scenarios.

Summarizing, our empirical results show that asymmetry indeed occurs in empirical data, especially in instable market situations, and should hence be incorporated in future copula models in finance.

\section{References}

[De1] P. Deheuvels: La fonction de dépendance empirique et ses propriétés. Un test non paramétrique d'indépendance, Acad. Roy. Belg. Bull. Cl. Sci. 65, 274-292 (1979).

[De2] P. Deheuvels: A Kolmogorov-Smirnov type test for independence and multivariate samples, Rev. Roumaine Math. Pures Appl. 26, 213-226 (1981).

[DeSS] H. Dette, K.F. Siburg, P.A. Stoimenov: A copula-based nonparametric measure of regression dependence, Scand. J. Stat. 40, 21-41 (2013).

[DuSS] F. Durante, S. Saminger-Platz, P. Sarkoci: Regular patchwork for bivariate copulas and tail dependence, Comm. Statist. Theory Methods 38, 2515-2527 (2009).

[FRW] J.D. Fermanian, D. Radulovic, M. Wegkamp: Weak convergence of empirical copula processes, Bernoulli 10, 847-860 (2004). 


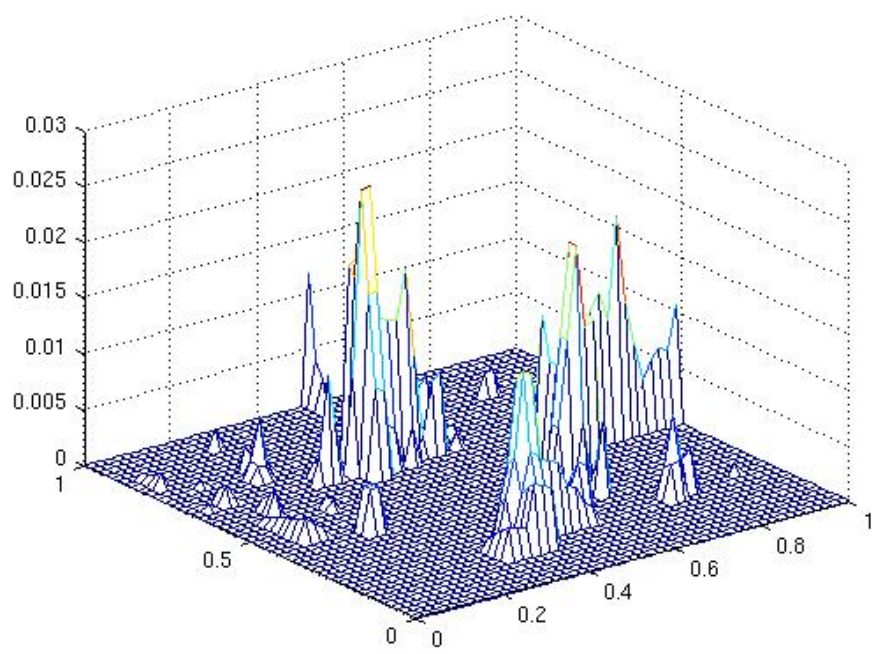

Figure 1: S\&P 500 versus VIX in 2006.

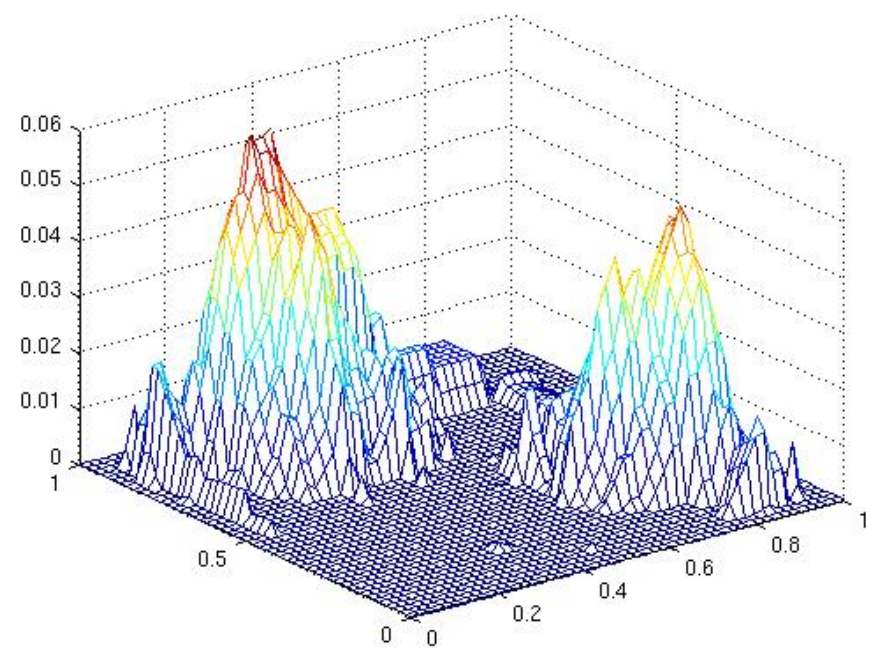

Figure 2: S\&P 500 versus VIX in 2009. 


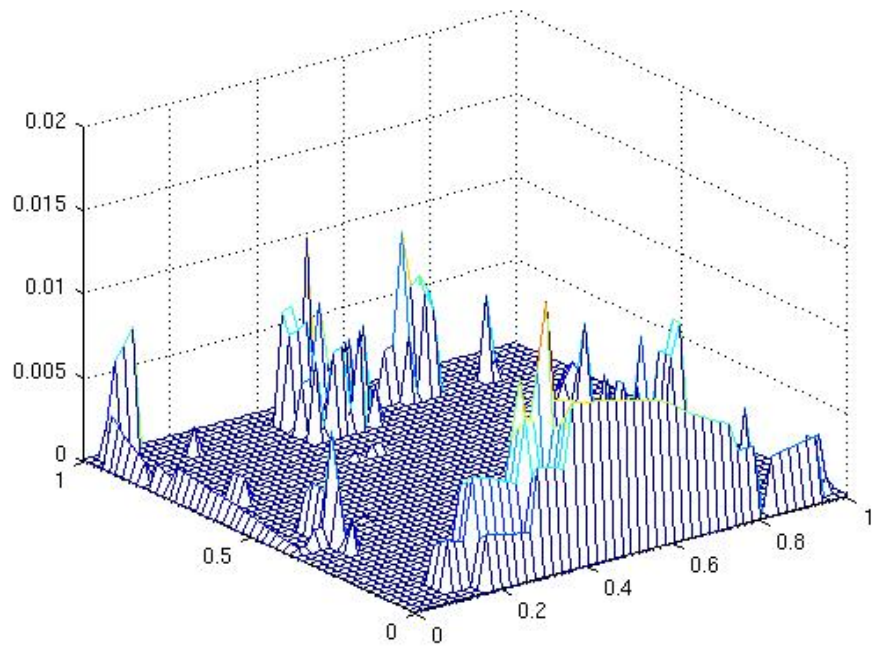

Figure 3: VIX versus HUI in 2006.

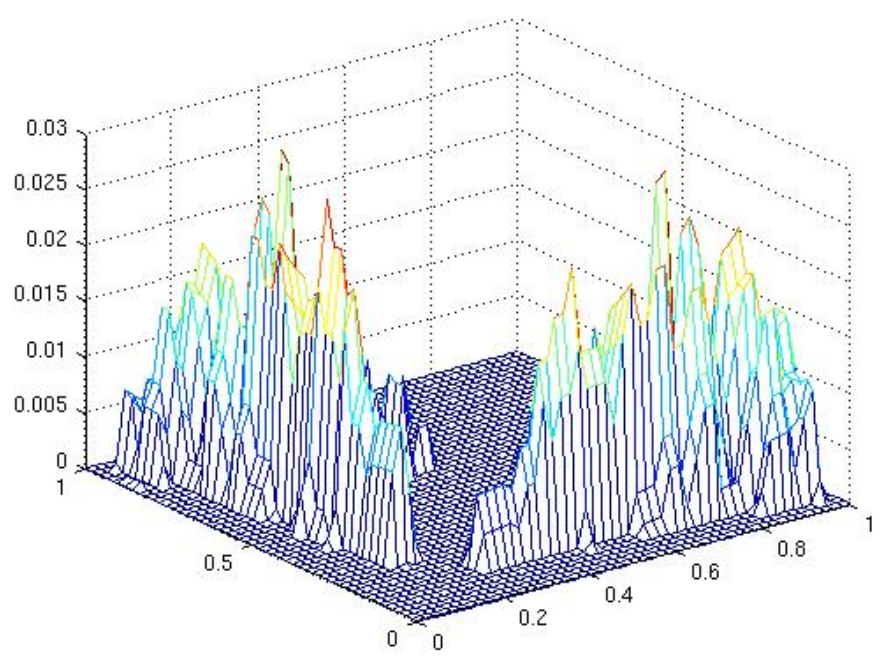

Figure 4: VIX versus HUI in 2009. 


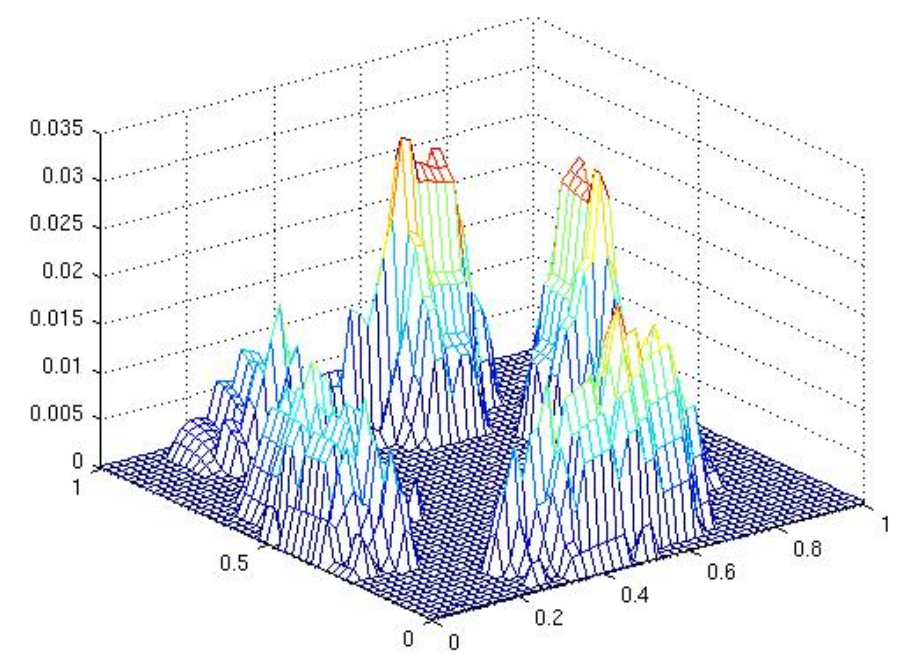

Figure 5: S\&P 500 versus HUI in 2006.

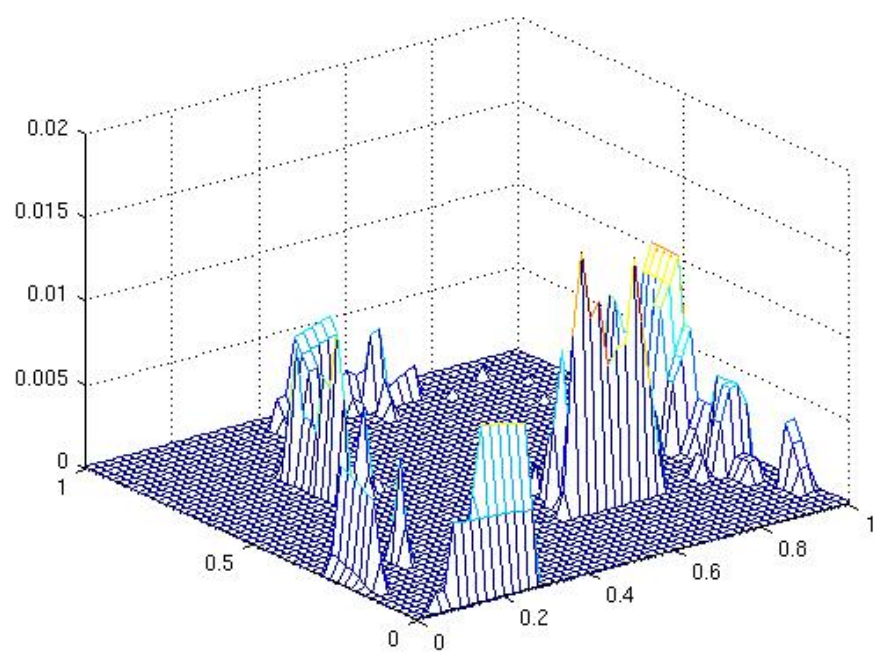

Figure 6: S\&P 500 versus HUI in 2009. 
[GS] P. Gänßler, W. Stute: Seminar on empirical processes, DMV Seminar 9, Birkhäuser Verlag, Basel, 1987.

[GKNY] C. Genest, I. Kojadinovic, J. Neslehová, J. Yan: A goodness-of-fit test for bivariate extreme-value copulas, Bernoulli 17, 253-275 (2011).

[GNQ] C. Genest, J. Neslehova, J.-F. Quessy: Tests of symmetry for bivariate copulas, Ann. Inst. Statist. Math. 64, 811-834 (2012).

[KM] E.P. Klement, R. Mesiar: How non-symmetric can a copula be?, Comment. Math. Univ. Carolin. 47, 141-148 (2006).

[KY] I. Kojadinovic, J. Yan: A non-parametric test of exchangeability for extremevalue and left-tail decreasing bivariate copulas Scand. J. Stat. 39, 480-496 (2012).

[Ne1] R. Nelsen: An Introduction to Copulas, 2nd edition, Springer 2006.

[Ne2] R. Nelsen: Extremes of nonexchangeability, Statist. Papers 48, 329-336 (2007).

[Rü] L. Rüschendorf: Asymptotic distributions of multivariate rank order statistics, Ann. Statist. 4, 912-923 (1976).

[SS] K.F. Siburg, P.A. Stoimenov: Gluing copulas, Comm. Statist. Theory Methods 37, 3124-3134 (2008).

[Skl] A. Sklar: Fonctions de répartition à $n$ dimensions et leurs marges, Publ. Inst. Statist. Univ. Paris 8, 229-231 (1959). 

